\title{
Amniotic Fluid - A Safe Alternative for the Treatment of Erectile Dysfunction
}

\author{
Michael P Zahalsky*, Gina Dessources, Melissa Marchand, Kelly Sooklal and Jonathan Swill \\ Department of Urology, Z Urology, 5850 Coral Ridge Drive Suite 106, Coral Springs, FL, 33076, USA
}

*Corresponding author: Michael P Zahalsky, Department of Urology, Z Urology, 5850 Coral Ridge Drive Suite 106, Coral Springs, FL, 33076, USA, Tel: 954-714-8200, Fax: 954-840-2626; E-mail: DrZ@zurology.com

Received: 12 Dec, 2017 | Accepted: 25 Jan, 2018 | Published: 31 Jan, 2018

Citation: Zahalsky PM, Dessources G, Marchand M, Sooklal K, Swill J (2018) Amniotic Fluid - A Safe Alternative for the Treatment of Erectile Dysfunction. Cell Stem Cells Regen Med 3(2): dx.doi.org/10.16966/24726990.117

Copyright: (C) 2018 Zahalsky PM, et al. This is an open-access article distributed under the terms of the Creative Commons Attribution License, which permits unrestricted use, distribution, and reproduction in any medium, provided the original author and source are credited.

\begin{abstract}
Background: Amniotic fluid has been used for centuries in several medical fields due to its inherent anti-scarring, anti-microbial and antiinflammatory properties. To date, the use of amniotic fluid has not been explored extensively in the field of urology in humans and this paper is the largest to report an in depth study of its use for treating male dysfunction.

Objectives: To retrospectively observe and evaluate the safety and quantity of ProFlo, an Amniotic Fluid (AF) product used for injections in the treatment of men with Erectile Dysfunction.

Design, Setting, Participants: This is a retrospective observational study performed at Z Urology in Coral Springs, Florida. 78 patients were used for the clinical trial and patients were injected initially with $0.1 \mathrm{cc}$ of Trimix followed by various aliquots of AF depending on physician preference and patient's condition. Patients with Erectile Dysfunction (ED) were injected intracavernosally at the base of the penis. Follow-up visits were conducted over a period of 6 months.
\end{abstract}

Results: Screening was evaluated using the International Index of Erectile Function (IIEF-5) Questionnaire and Penile Doppler instrumentation. There were no major complications.

Outcome Measurements and Statistical Analysis: There was a statistically significant improvement in IIEF score $(\mathrm{P}<0.001)$ and in Peak Systolic Velocity on Penile Doppler $(\mathrm{P}<0.001)$.

Conclusions: Results indicate that there have been no significant adverse effects and that amniotic fluid is safe to be used homologously on the genitourinary system. ProFlo appears to be a safe alternative therapeutic option for men with Erectile Dysfunction. More research is required to evaluate its effectiveness in the treatment of ED.

Patient Summary: The data indicates that Penile Doppler is an effective tool in evaluating PSV values before and after the ProFlo injections. This study shows ProFlo to be a viable candidate to treat male dysfunction with limited risk complications.

Keywords: Erectile dysfunction; Amniotic fluid; Peak systolic velocity

Abbreviations: ED: Erectile Dysfunction; AF: Amniotic Fluid

\section{Introduction}

ED is usually a micro vascular disease characterized by difficulty obtaining and/or maintaining an erection firm enough for intercourse. Aging has been associated with resistance in penile blood flow, possibly due to decreases in nitric oxide synthase-containing nerve fibers [1,2]. Erectile dysfunction is frequently associated with vascular risk factors such as diabetes mellitus, coronary artery disease, and hypertension [3]. In particular, $50 \%$ to $75 \%$ of men with diabetes mellitus have ED regardless of their age [3].

Current therapies for Erectile Dysfunction (ED) have proven to be effective however the side effects, long-term financial burden, and daily to pre-coitus regimen has proven to be a major deterrent for a large portion of men. These therapies can be as broad as changing one's lifestyle to oral medications, vacuum devices, injection therapy, and surgery. As a result, the demand for a non-pharmaceutical approach to treating these conditions has been growing, leading to the exploration and investigation of alternative therapies.

In 2015 and 2016, Levy et al, first reported the feasibility of using stem cells to treat human patients with ED $[4,5]$. Both studies suggested that the stem cell approach showed promise and could be effective as a non surgical treatment for patients $[4,5]$.

In the present study, we report on the use and safety profile of Amniotic Fluid. Amniotic fluid has been around since the early 1900s since scientists saw its potential for applications in treating wounds. Today, amniotic fluid is becoming more conventional and is being used where inflammation is prevalent such as pain management, surgeries, dentistry and various orthopedic areas [6-9]. Amniotic fluid is derived from live birth cesarean sections and contains multiple growth factors and interleukins. Amniotic tissue is readily available, as it is often discarded after childbirth. The use of this tissue poses no added risk to the fetus or mother, eliminating the ethical concerns associated with obtaining embryonic stem cells. Amniotic tissue is comprised of an extracellular matrix, which acts as a natural scaffold for cellular attachment and structural support for cells as well as collagen types I, III, IV, 
V, and VI, hyaluronic acid, and a host of growth factors [10]. In addition, it possesses antimicrobial properties, including beta-defensins [10]. Some of these growth factors have been individually studied and shown to help in erectile dysfunction and to reduce inflammation, such as Stromal Derived Factor 1 and Interleukin 6 [11]. Despite the fact that Amniotic Fluid has been used for years in various medicinal fields, we are the first to explore the safety and quantity that can be used in Urology for ED. According to the FDA draft guidance from December 2014, AF is considered a nonstructural tissue that has a metabolic or biochemical role in the body. AF is from the genitourinary system, and therefore it is able to be used for homologous use on the genitourinary system. Since AF has no dosage, our team looked retrospectively at safety related to injections and quantity of AF used during injections.

\section{Methods}

Amniotic Fluid injection may be a hopeful, safe and effective treatment for erectile dysfunction. ProFlo is amniotic fluid from live birth Cesarean Sections that is minimally manipulated and procured from a FDA cleared tissue bank, Vivex. It meets all the criteria to be regulated under the Public Health Service Act section 361. ProFlo was used for the frozen allograft for these studies and vials of $1.0 \mathrm{cc}$ to $2.0 \mathrm{cc}$ were utilized for the procedures. Vivex's processing methodologies are able to preserve the natural content and growth factors of the AF. The $\mathrm{AF}$ is a $100 \%$ natural, protein rich fluid that contains multiple growth factors and cytokines that aid in healing primarily by its anti-scarring, anti-microbial, and anti-inflammatory properties [7]. Since these growth factors play a critical role in wound healing, we tend to believe that they may eventually restore blood flow to the penis and assist in vasculogenesis. First, informed consent was received from all 78 patients. Inclusion criteria included patients who had ED and did not want to a penile prosthesis. These patients suffered from chronic ED for at least 6 months and had tried several alternatives prior to biologics. The average age of patients that participated in this study was 58 years where the youngest was 30 and the oldest was 81 years. Patients with known penile trauma or penile pathology were excluded from this study.

Prior to injections, patients filled out the International Index of Erectile Function (IIEF-5) to obtain an initial assessment of the severity of their ED. Penile Doppler and Ultrasonography was used to measure Peak Systolic Velocity (PSV) and End Diastolic Velocity (EDV) in the ED patients. All data was collected using the GE Logiq Ultrasonographer with an 11 megahertz probe. All patients were initially injected with $0.1 \mathrm{cc}$ of Trimix for standardization. The trimix (30-4-50) composition was comprised of $30 \mathrm{mg}$ of papaverine, $4 \mathrm{mg}$ of phentolamine, and $50 \mu \mathrm{g}$ of prostaglandin. PSV was measured 20 minutes post Trimix injection, both pre and post AF injection. Dosage quantities of AF varied between $1.0 \mathrm{cc}$ and $2.0 \mathrm{cc}$ depending on the physician's preference and patient's condition. Patients with ED were injected intracavernosally at the base of the penis.
Follow-up appointments were scheduled over a period of 6 months. Prior to initiating the study, patients provided consent to continue to monitor their medical profile and collect any data needed. Apart from consulting with patients about their reaction and response to the injections, safety based questions were also utilized to assess the risk of using biologic products. Additional questions were asked that mimicked the Xiaflex complication product insert chart / questionnaire [12].

\section{Results and Discussion}

78 patients were injected with ProFlo AF. 41 patients injected with AF had pre-post PSV and 33 patients had pre-post IIEF-5 scores. Only PSV values were collected and analyzed for this study. It was the most significant method that we used as an indicator of arteriogenic impotence. In the flaccid state, the systolic and diastolic flow is absent or at a minimum. Once the penis is erect, pressure increases with the onset of the erection and the systolic and diastolic flows also increase. Figure 1 shows the before and after PSV values obtained from the 41 patients with the standard error for all values. The average PSV after Trimix injection before administering AF was $25.53 \pm 10.39$ $\mathrm{cm} / \mathrm{s}$ (standard error 1.62) whereas after a period of 6 months the average PSV observed was $42.43 \pm 15.11 \mathrm{~cm} / \mathrm{s}$ (standard error 2.36). This resulted in an average increase of $16.90 \mathrm{~cm} / \mathrm{s}$ post injection of the amniotic fluid. A two tailed t-test assuming unequal variances indicated that there was a significant difference and our p-value $<0.001$. Similarly, an increase was observed in the IIEF score for the group figure 2. IIEF scores ranged from 5 to 23 before the initial AF injection and were between 7 to 25 after AF injection. The average IIEF score pre injection was $10.06 \pm 5.1$ (standard error 0.89) and after injection was $14.4 \pm 5.2$ (standard error 0.90). Likewise, our p value obtained from the two tailed was significant, $\mathrm{p}<0.001$. The average difference in change from the pre-post injection is 4.40 . The patient's only complaints were of minimal bruising and pain at the injection site for 2-7 days after injections. Table 1 shows the records of any complications observed over the 6 months for the patients undergoing the clinical study. The quantity of ProFlo did not appear to have a relationship to the frequency of complications. Pain during procedure and penile ecchymosis for less than one week were frequently noted.

Table 1: Record of complications related to AF injections.

\begin{tabular}{|l|c|l|c|}
\hline COMPLICATIONS & AMOUNT & COMPLICATIONS & AMOUNT \\
\hline Corporal Rupture & 0 & Vesicles at Injection Site & 0 \\
\hline Penile Fracture & 0 & New Onset of ED & 0 \\
\hline Penile Hematoma & 0 & Worsening of ED & 0 \\
\hline Penile Infection & 0 & Skin Discoloration & $1(2.56 \%)$ \\
\hline Penile Swelling & 0 & Localized Edema & 0 \\
\hline Penile Pain & 0 & Dyspareuina & 0 \\
\hline Blood Blister & 0 & Injection Site Pruritus & 0 \\
\hline Genital Pruritus & 0 & Suprapubic Pain & 0 \\
\hline Painful Erection & $2(2.56 \%)$ & & \\
\hline
\end{tabular}




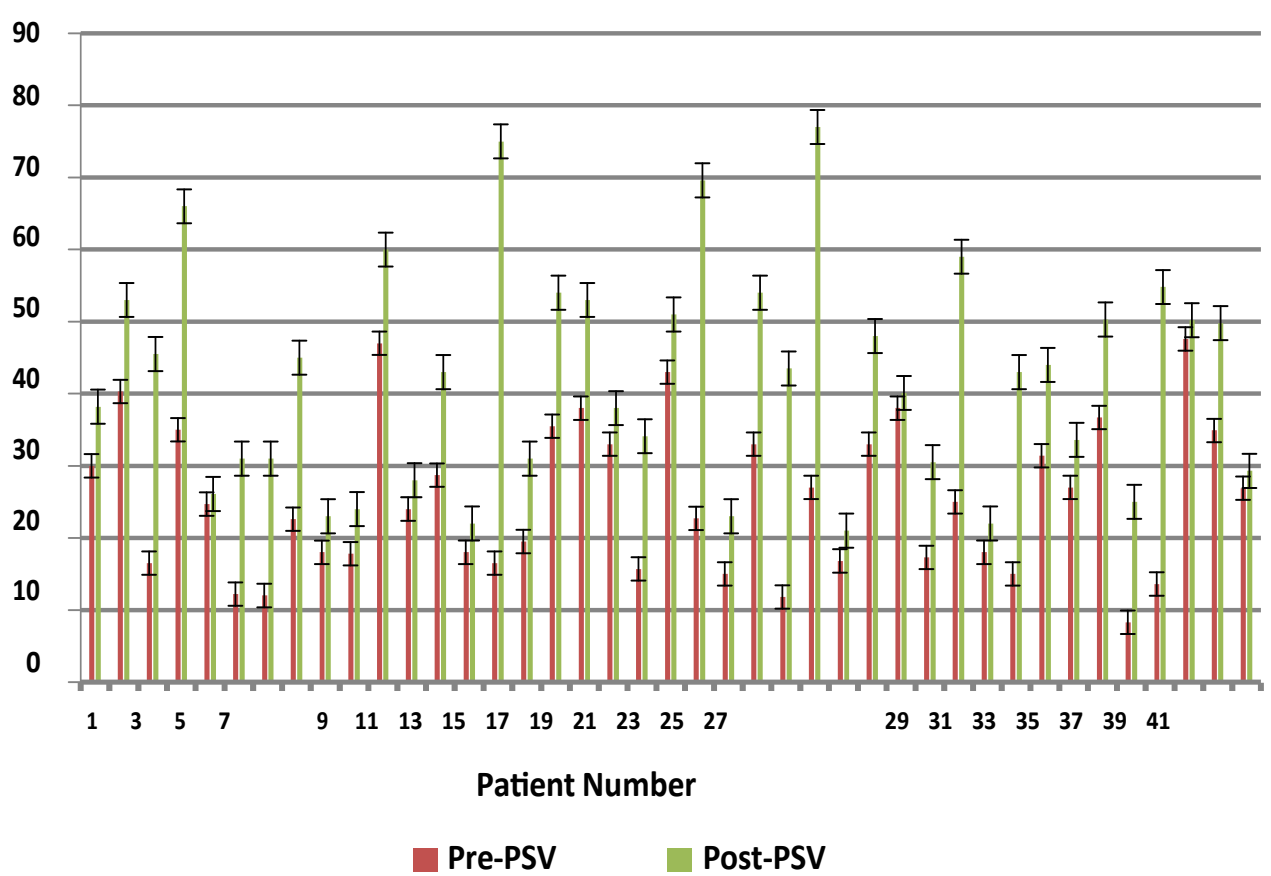

Figure 1: Pre and post PSV data for patients injected with ProFlo.

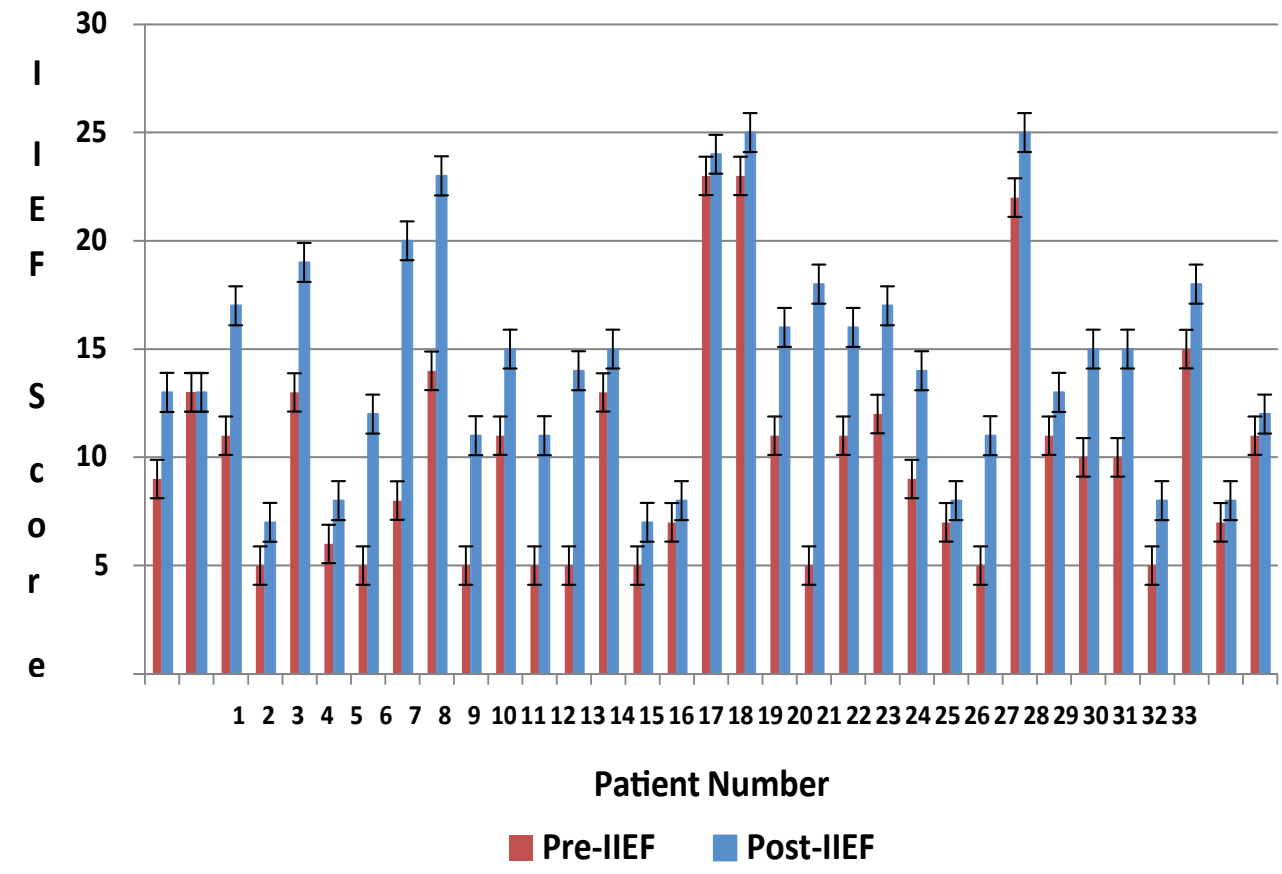

Figure 2: Pre and post IIEF values.

Approximately $80 \%$ of patients complained of procedural pain lasting 2-5 days that required over the counter pain medication such as Tylenol. One patient reported ecchymosis lasting longer than 5 days after one of their injections (2.8\%). Out of all 78 patients, there were no reports of penile hematoma. During future follow-up visits, patient responses were encouraging on the screening based safety questions. Typically when Hepatitis C Virus (HCV) is contracted about $75 \%$ of individuals typically show no symptoms when they first contract an infection. The remaining $25 \%$ show varying symptoms of fatigue, loss of appetite, muscle aches or fever. The patients questioned had no signs of HCV. Likewise, patient's responses reflected no indications of early symptoms to the HIV virus and several individuals stated that they were content with the ProFlo injections. During the course of this retrospective study, there were no reported cases of transfusion reaction, acute and/or chronic rejection of ProFlo. There were also no reported cases of Blood Born Pathogen Transmission, such as HIV or Hepatitis 
C, however no pretreatment screening or post treatment screening was conducted. No cancers were newly diagnosed in the patients injected. There has never been a documented case of Blood Borne pathogen transmission from the University of Miami Tissue Bank.

Our present study shows very promising results for the treatment of erectile dysfunction with amniotic fluid. Even though, the study lacks comparison of other intracavernosally and oral drugs to ProFlo, the increase in PSV in the 41 patients maysuggest that erectile dysfunction was improved by improving arterial flow. The Statistically significant improvement in IIEF score, demonstrates that patients' erections improved enough for them to be satisfied with the improvements in PSV. We believe, this correlation is very encouraging for the future of ED. Amniotic fluid has been shown to exert an anti-inflammatory effect by inhibiting inflammation [13]. Silini et al have reported that the amniotic tissue inhibits cytokines such as tumor necrosis in the presence of dendritic cells, as well as inhibiting transforming growth factor-beta, interleukin- 8 and fibroblast proliferation [13]. These findings indicate that amniotic tissue has the ability to dampen the "cytokine storm" that occurs after an injury in an adult, which would lead to beneficial impacts on healing and scar formation [13]. In the case of ED, the growth factors present in the amniotic fluid we hypothesize maybe used to repair, restore, and regenerate blood vessels to and within the corpora of the penis as well as the corpora themselves to improve erections.

\section{Conclusions}

This is the largest and first documented study in literature on the safety of using ProFlo, amniotic fluid to treat ED in humans. Based on the data accumulated, there have been no significant adverse effects found in the 78 patients. Patients were evaluated based on PSV and IIEF data. After 6 months from their initial injection, the patients PSV and IIEF values both increased indicating that the amniotic fluid treatment was effective in improving their erectile function. A longer time period is necessary to ascertain longevity effects of the biological injection but these initial studies indicate that this treatment can help increase penile blood flow and improve erectile dysfunction. ProFlo appears to be a safe alternative treatment option for men with ED. This is a retrospective study on the safety and quantity of ProFlo used or to be able to use to treat humans with ED. Further research studies are underway to evaluate ProFlo Amniotic Fluid's efficacy in treating ED patients.

\section{References}

1. Carrier S, Nagaraju P, Morgan DM, Baba K, Nunes L, et al. (1997) Age decreases nitric oxide synthase-containing nerve fibers in the rat penis. J Urol 157: 1088-1092.

2. Mangir N, Akbal C, Tarcan T, Simsek F, Turkeri L (2014) Mesenchymal stem cell therapy in treatment of erectile dysfunction: autologous or allogeneic cell sources? Int J Urol 21: 1280-1285

3. Lewis RW, Fugl-M KS, Corona G, Hayes RD, Laumann EO, et al. (2010) Definitions/epidemiology/risk factors for sexual dysfunction. J Sex Med 7: 1598-1607.

4. Levy JA, Marchand M, lorio L, Zribi G, Zahalsky MP (2015) Effects of Stem Cell Treatment in Human Patients With Peyronie Disease. J Am Osteopath Assoc 115: e8-e13.

5. Levy JA, Marchand M, lorio L, Cassini W, Zahalsky MP (2016) Determining the Feasibility of Managing Erectile Dysfunction in Humans With Placental-Derived Stem Cells. J Am Osteopath Assoc 116: e1-e5.

6. Bennett NT, Schultz GS (1993) Growth factors and wound healing: biochemical properties of growth factors and their receptors. Am J Surg 165: 728-737.

7. Temple HT, Malinin TI (2016) Orthobiologics in the Foot and Ankle. Foot Ankle Clin 21: 809-823.

8. Maraldi T, Riccio M, Pisciotta A, Zavatti M, Carnevale G, et al. (2013) Human amniotic fluid-derived and dental pulp-derived stem cells seeded into collagen scaffold repair critical-size bone defects promoting vascularization. Stem Cell Res Ther 4: 53.

9. Werber B, Martin E (2013) A prospective study of 20 foot and ankle wounds treated with cryopreserved amniotic membrane and fluid allograft. J Foot Ankle Surg 52: 615-621.

10. Buhimschi $I A$, Jabr $M$, Buhimschi $C S$, Petkova $A P$, Weiner $C P$, et al. (2004) The novel antimicrobial peptide beta3-defensin is produced by the amnion: a possible role of the fetal membranes in innate immunity of the amniotic cavity. Am J Obstet Gynecol 191: 1678-1687.

11. Sopko N, Matsui H, Kates M, Lough D, Liu X, M, et al. (2016) Stromal Derived Factor-1 treatment augments nerve regeneration via CXCR4 activation of neurotrophic factors in the major pelvic ganglion in a dose-dependent fashion. J O U 195: 1139-e1140.

12. (2016) Efficacy proven in 2 Large Multicenter Studies with Xiaflex $^{\circ 1}$.

13. Silini A, Parolini O, Huppertz B, Lang I (2013) Soluble factors of amnion-derived cells in treatment of inflammatory and fibrotic pathologies. Curr Stem Cell Res Ther 8: 6-14. 